\title{
MONITORING LURIGANCHO-CHOSICA, PERÚ LANDSLIDEUSING INSAR TECHNOLOGY
}

\section{LUCERO RODAS, JORGE HUARNIZ, CARMEN VILLON, RODOLFO MORENO, WILVER AUCCAHUASI}

Comisión Nacional de Investigación y Desarrollo Aeroespacial del Perú, Lima, Perú

\begin{abstract}
From January14th to March 11th 2017, constant rainfall produced by El Nino Phenomenon has suffered the capital of Lurigancho district, Chosica, located in Lima Peru. Due to heavy rains, in the period from January to March, many landslides occurred in differentstreams of Chosica, Huampani, Chacrasana, Pedregal, Quirio, Carosio, Corrales, Rosario, Mariscal Castilla Santo Domingo, California, and La Cantuta, ravines wereactivated, the first landslide occurred on January 14th, and the second one, occurred from March 15th to March 19th of the year 2017. This rainstorm not only caused landslides, but also caused overflow of the Rimacriverthataffected 6,058 people, 2,015 homes and $10.75 \mathrm{~km}$ communication routes. This studyisfocused on the Application of Persistent ScattererInterferometrySynthetic Aperture Radar (PSInSAR) Technique for mapping displacement produced by landslides. This research has been used Sentinel-1 SAR images, SLC 17 scenes in ascending orbit and 26 scenes in descending orbit, from October of 2014 to December of 2016. This study has been workedwithPSInSAR Technique, based in Time seriesInSAR, consideringChosicaLandslide as a debris flows with very slow displacement, this technology has used to acquire the deformation prior in the Line of sight direction (LOS). First, the displacement result in ascending and descending orbit has been obtain by processing with ENVI 5.3-Sarscape tool, it has applied Persistent Scaterrermethod. Second, Displacement result in descending orbit, has been compared and analyzed with the slipped areas after landslide in 2015 and 2017 identified using aerial photo provided by DIVRA (Direction of surveillance and aerial recognition). Posteriorly both orbits were combined spatially with the intention of getting Vertical (Up-Down) and Horizontal (East-West) DisplacementusingArcGis-Python tools.It maybe more useful to compare the result of the displacement using the PSI InSAR technique with the GPS technique, which would provide and test the accuracy of the results obtained by PSI. Could be ideal to use a DEM whose spatial resolution in meters can reached less than $30 \mathrm{~m}$, nevertheless SRTM $30 \mathrm{~m}$ can be productively exploited. It is advisable to plant more treesaround the Chosica'sstreams, whichwill minimize the damage and protect the population and, since there is not much vegetation, when the rains appear and find the land devoid of it, the terrain loses stability and it produces landslide.
\end{abstract}

KEYWORDS: Lurigancho-Chosica, Landslide, Time Series Insar, Psinsar, LOS, Displacement

Received: Jun 08, 2020; Accepted: Jun 28, 2020; Published: Sep 25, 2020; Paper Id.: IJMPERDJUN20201419

\section{INTRODUCTION}

Almost all the cities in Perú are vulnerable by rains, floods, landslides, hails, thunderstorms and snowfalls.Landslide monitoring usingInSAR Technology aims to provide high quality information that allows knowing the predisposition that a natural eventoccurs in eachgeographicspace.The measurement of landslidedisplacement often represents the most efficient method for defining its behavior, allowing one to observe the relationship with unchaining factors and to assess the effectiveness of the mitigation measures. Persistent ScattererInterferometry (PSI) represents a potent tool to measure landslide displacement, as it offers a clear view 
that can be repeated at different time intervals and at various scales. This study pretend integrated PSI method with photogrammetric interpretation of slipped areas, since the joint use of satellite and airborne data facilitates a better understanding of landslide geometry and kinematics

The present work seeks to make an analysis of the aspects that involve the development of landslide in Lurigancho-Chosica district, an area between the coordinates: Southwest point $\left(-12^{\circ} 3^{\prime} 0\right.$ "S $-76^{\circ} 51^{\prime} 0$ " W; Northeast point $\left(-11^{\circ} 51^{\prime} 0\right.$ "S $\left.-76^{\circ} 36^{\prime} 0 " \mathrm{~W}\right)$, shown in Figure 1.

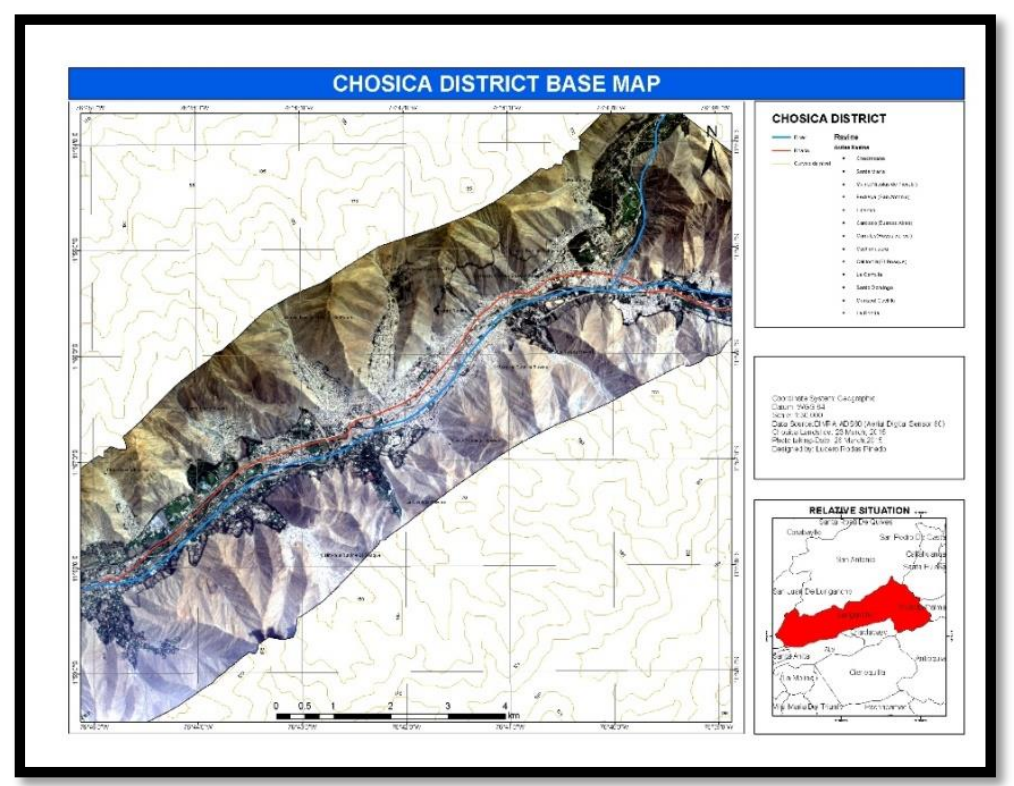

Figure 1 : Chosica District. Aerialphotographytaken by DIVRA ADS80. Jan 172017

It is important to understand and respond hypotheses raised in this study, as a first hypothesis: which phase of landslide can be identify in the deformation result obtained by INSAR technology, considering three phases, first step is when the land block (origin area) break produced by hard rainfall, then when the debris flows in ravine and finally when the mud flows and spreads throughout the nearby area. As a second hypothesis: There is a probability to find a prior deformation before to the landslide occurred in 2017, because the interval of InSAR data has been obtained in the period 2014-2016, notice that on February 09th and March 24th of the year 2015 triggered a landslide in Lurigancho-Chosica[1]. If so, this study can verify and compare by temporal visual interpretation map using 2015-2017 aerial photo data provided by DIVRA. This analysis can help us to take precautions for any future landslides.

It has to be mention, beforelandslide on 2017, there were other small landslide occurred on February 09th 2015 and on March 23th 2015, these landslides has to be consider in the results, 535 people affected, 496 affected houses reported by INDECI and COEN.[2]

\begin{tabular}{|l|c|c|}
\hline & Feb-March 2015 & 15 January 2017 \\
\hline Affected people & 535 & 6,058 \\
\hline Affected houses & 496 & 2,015 \\
\hline Affected & - & 10.75 \\
\hline
\end{tabular}

Figure 2 : Preliminary Evaluation of damages 
For all the aforementioned, this research scheme will seek to answer the following general question: Is PSInsar technology useful for monitoring areas and detect possible landslides? To achieve our objectives, we will develop the background and theoretically define the variables of our research. Finally, after addressing the methodological framework, we will analyze the displacement maps where we will obtain precise information that will facilitate the correct landslide monitoring in Chosica.

\section{METHODS Y MATERIALS}

\section{AREA DEFORESTED BY ILLEGAL MINING}

In thisstudy, it has been chosen Sentinel-1 A/B SAR images, in Slant Look Interferometric Wide Swath(IW) mode; Data products are available in dual polarization and single polarization, this research was selected VV polarization; to analyze displacement, Level-1 Single Look Complex products was selected. Sentinel Data has downloaded from Open Access Hub (https://scihub.copernicus.eu/dhus/\#/home). The time interval of those images are from October of 2014 to December of 2016, as shown in the following data:

- 26 scenes of descending orbit Sentinel-1A/B SAR images

- 17 scenes of ascending orbit Sentinel-1A/B SAR Images

- POD PreciseOrbitEphemerides,

- Aerial photo by ADS80 Sensor of Landslide March 2015, Source: DIVRA

The chosen method can be summarized in the following workflow, as shown in figure 2

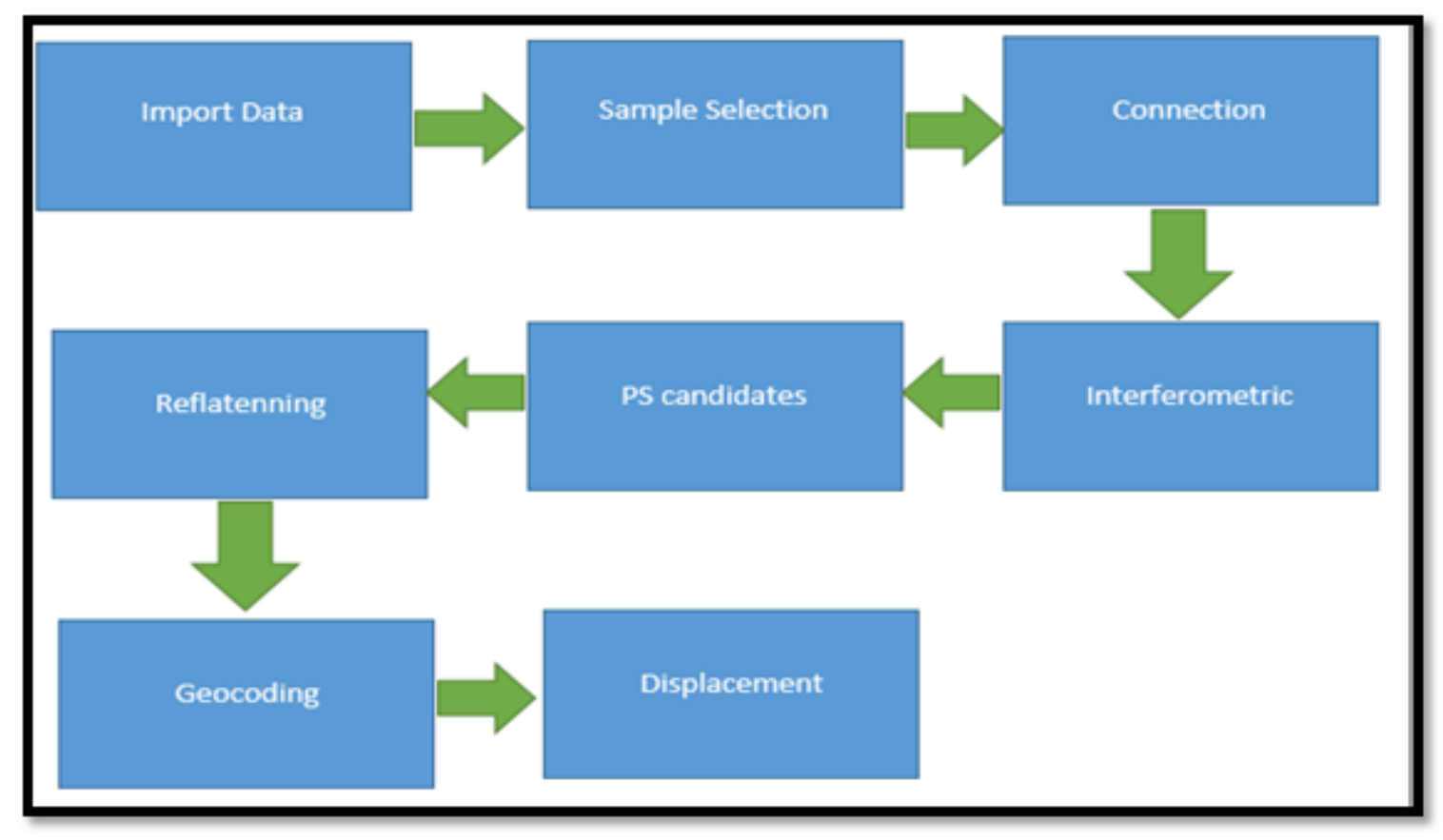

Figure 3 : Proposal block diagram

It has to count that beforestart Persistent Scaterrer workflow, Descending (.manifest) data was import together with POD PreciseOrbitEphemerides for Sentinel data. There are severaladvantages of using precise orbits to improve the interferometry processing, especially in relationship with the removal of orbital related fringes in the interferogram. 


\section{CONNECTION GRAPH AND MASTER SELECTION}

For PInSAR Method. Sentinel dataset contains 26 scenes in descending and 17 scenes in ascending acquisitions IW mode and VV polarization. Table 3 shows the temporal coverage from October 2014 till December 2016 images, it has selected as Persistent Scatterers Master image for descending orbit:2015/11/05 and for ascending orbit: 2016/04/22; and Normal baseline values are shown respectively.

\section{INTERFEROMETRIC PROCESS}

This functionality carries out in an automatic way, the followingprocessingsteps: Coregistration, InterferogramGeneration, Flattening and Amplitude dispersion index.The purpose of thisstep is to get the flattenedinterferogram, where the constant phase (due to the acquisition geometry) and the topographic phase (SRTM DEM provided) have been removed. This step alsoperformed the coregistrationwith DEM.

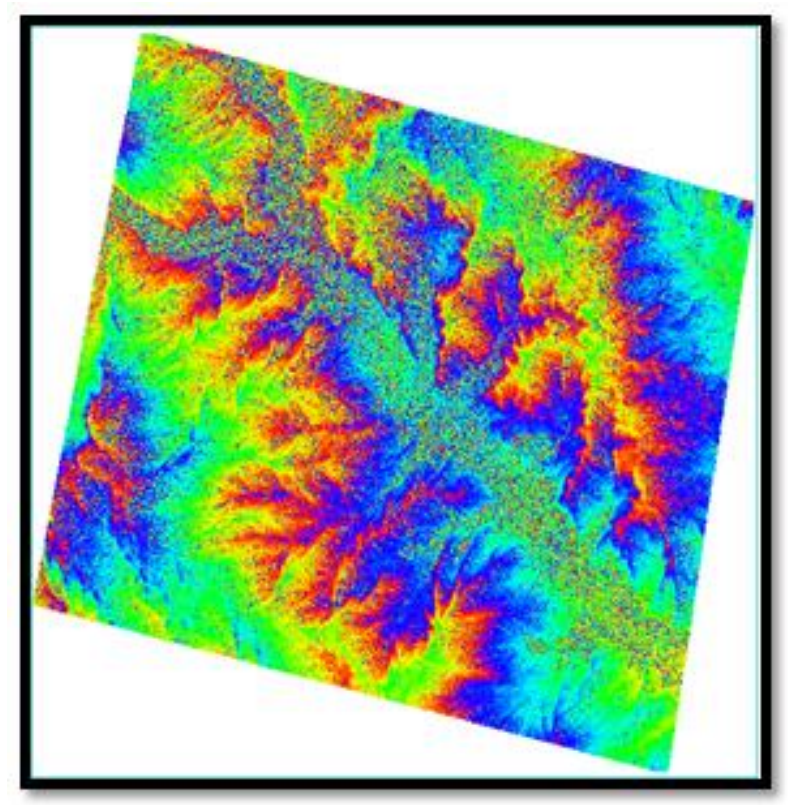

Figure 4 : DifferentialInterferogram of 2014/10/17 with Master image

\section{CORREGISTRATION STEP}

All sampled images are coregistered over the resampled "Master file". Range direction oversampling looks 5.

\section{THE INTERFEROGRAM FLATTENING}

Is execute using an input reference the Digital Elevation Model. If it's better DEM accuracy/resolution, it will be the better the result in terms of topography removal. In this research has been choose Shuttle Radar Topography Mission (SRTM) at a resolution of 1 arc second (approximately 30 meters).

\section{THE AMPLITUDE DISPERSION INDEX (D)}

To get smaller values of amplitude dispersion, it will generate very strong amplitude pixels (urban areas), the phase information of the solid signal pixels are expected to be more stable. Likewise, this research are interested to increase the SNR (signal to Noise Ratio) to supply a more credible coherence estimation. Hence, thresholding on the dispersion index is a very practical way of selecting points that are expected to have the smallest phase dispersion 


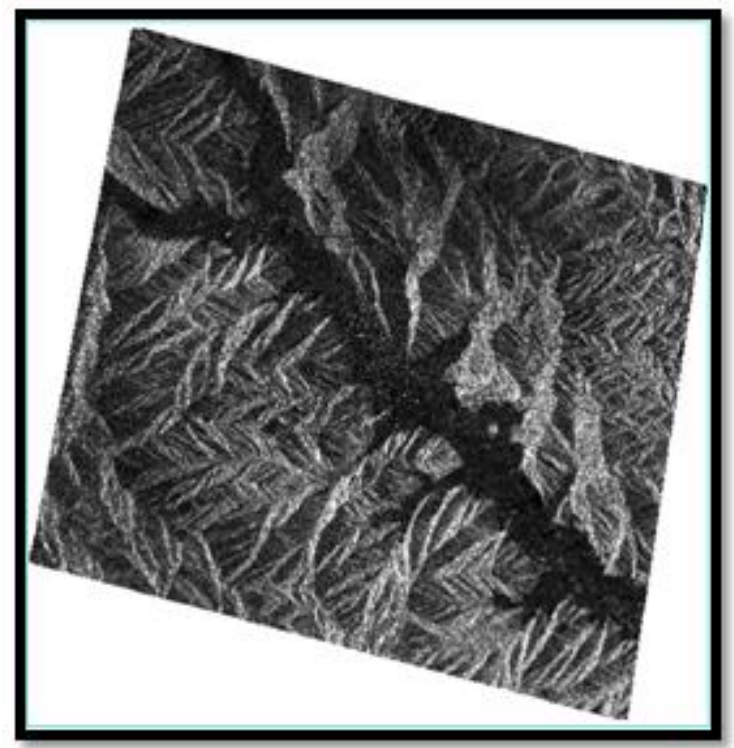

Figure 5 : Amplitude Dispersion Index (D)

Finally it's generated 25 interferometry stack for all slaves' images with the master image. After the interferogramsgeneration, an offset phase is removedfrom all interferograms.

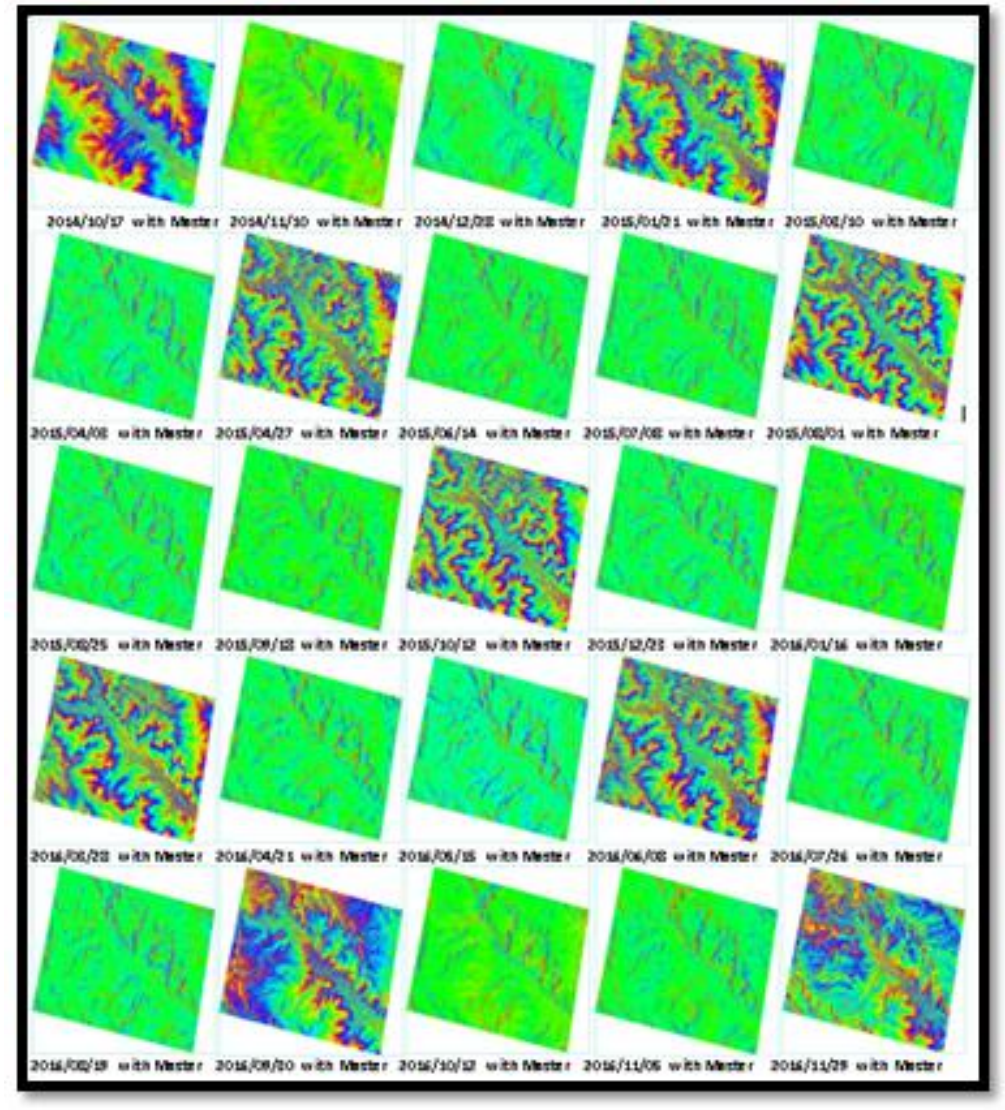

Figure 5 : Interferogramstacking

\section{PS CANDIDATES SELECTION}

WhereDispis the displacement at time $\mathrm{t}, \mathrm{V}$ is the displacement velocity.[5]. In this step the first displacementvelocity and 
heighterror are estimatedwithoutremovinganyatmospheric phase component.They are used to flatten the complexinterferograms. The number of the 'Reference Points' depends on the size of the Area of Interest, for this research, just one 'Reference Point' is selected for Areas within $5 \mathrm{~km} 2$. Good PS candidates can be roofs, poles, bridges, they are typically found in urban settlements, or other human made structures such as greenhouses, dams, metallic and concrete characteristics. Additionally, these artificial characteristics, also natural targets such as well-exposed outcropping rock formations are potential PS.

In figure 8 some value jumps can be noticed, represented by color blue. In figure 9, several value jumps can be noticed, however, in the overlap zones between the different blocks of "cc_first", simply the better coherence is taken. This can lead to value jumps between blocks. This effect is normal for coherence and should not be considered as an error, Moreover, Research's study area is limit, so these results can be used for the next step, and this small value jumps in the first model inversion results can be removed by the atmospheric filter.
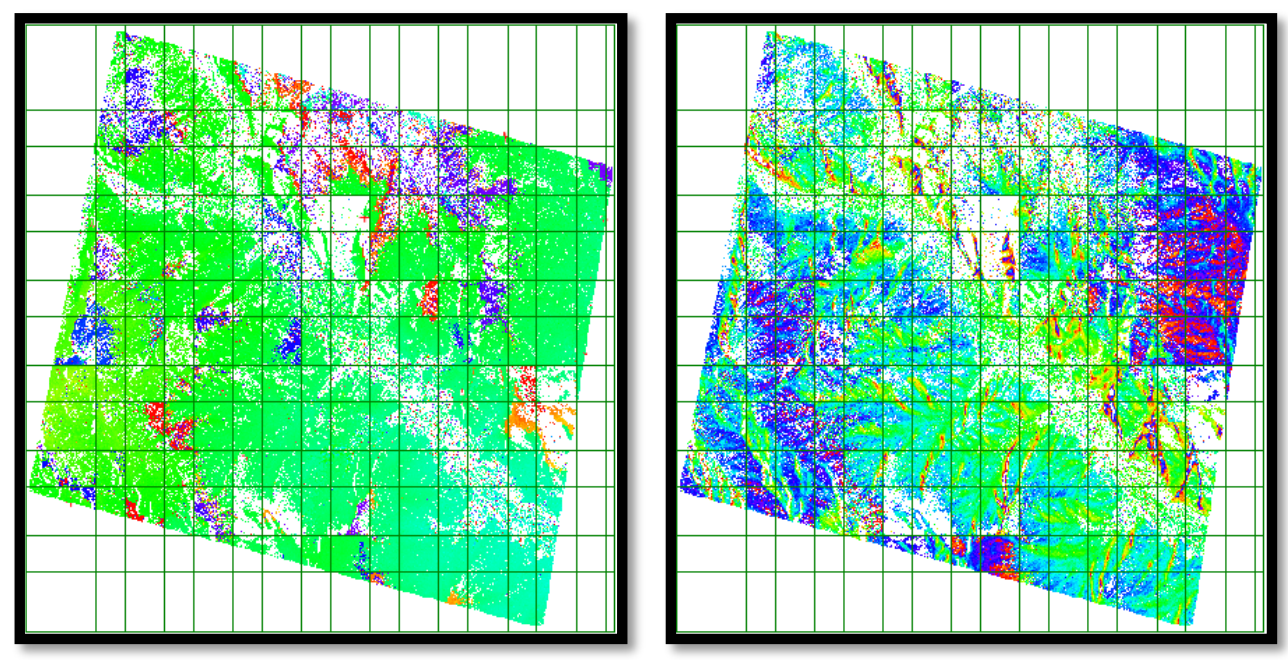

Figure 6 : Left: PS-Descending'sVelocity_first, after applying a color scale; right: PS-Descending'sHeigh_first, afterapplying a color scale

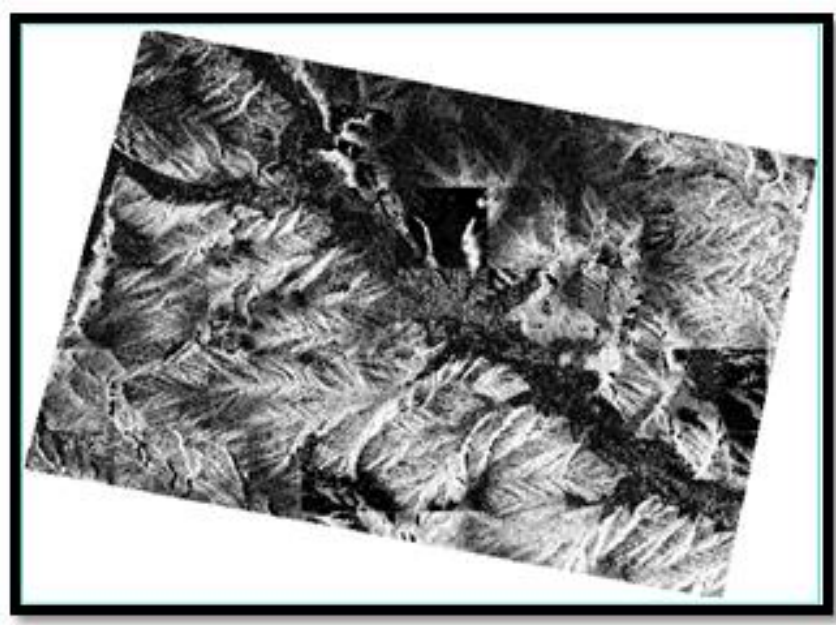

Figure 2 : PS-Descending'scc_first

\section{REFLATETENING GENERATION AND FILTERING ATMOSPHERE}

Atmospheric in homogeneities create an atmospheric phase screen (APS) superimposed on each SAR image that can 
considerably compromise accurate deformation monitoring. In effect, the APS exhibits a low-wavenumber spectral behavior (according to the atmospheric water vapor distribution in the troposphere) and cannot be detected and estimated from the coherence map associated with each interferogram.[6]

The atmospheric phase is obtained applying spatial low pass filter and temporal high pass filter, then the atmospheric phase are interpolated forming the Atmospheric phase screen (APS), as showing in the figure 10.

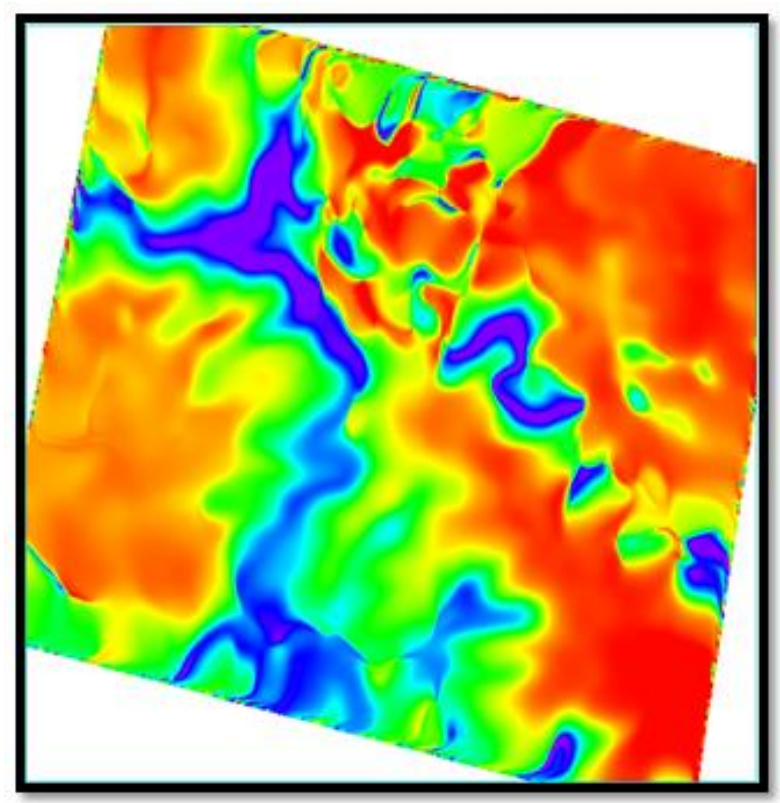

Figure 3 : EstimatedAtmosphere Phase Screen relative to the Sentinel SAR image acquired on October17th, 2014

\section{COHERENCE THRESHOLDING AND GEOCODING}

The PS products are geocoded and the displacements has generate shape file (points) as result. For this research was selected as input product coherence threshold(0.80) that means that Pixels with coherence values smaller than this threshold cannot be kept as Persistent Scatterer. [5]Finally, the interferogrambecomesequal to the displacement or deformation.
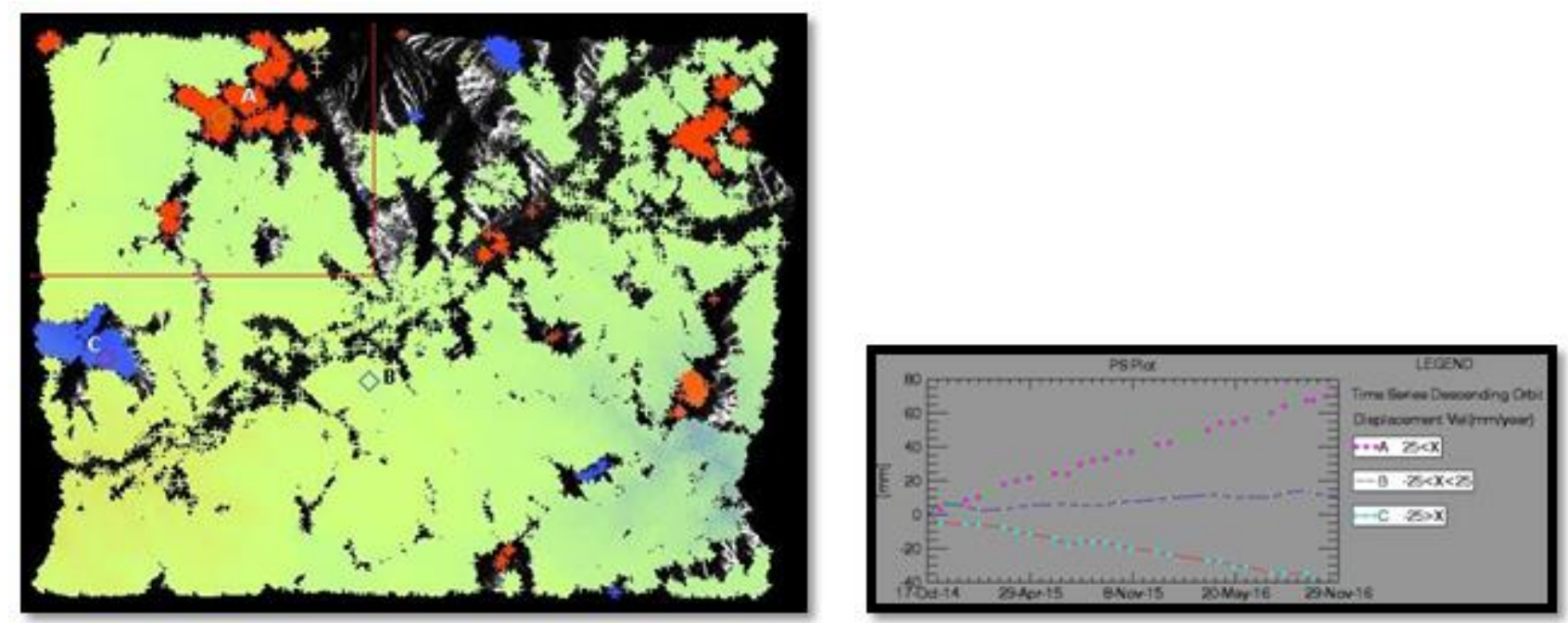

Figure 11: PS-Descending orbit results for velocity displayed on the Mean Geo intensity image 


\section{RESULTS}

\section{Displacement map-Descending Orbit Results}

The time-seriesdescendingdisplacementresults before occurs Landslide occurrence are obtained. Figure 14 show the Displacementmap of Lurigancho-Chosicawhere, the dotted black polygon represents the area of Landslide damage; the orange and red polygons represent the slipped area detected using aerial photos respectively. Note, as the deformation is into the line-of-sight (LOS) direction, the red color points indicates movement towards the sensor and the blue color points indicates movement away from the sensor. In the other hand, the orange and red polygons for each displacement map represent slipped areas detected using optical remote sensing images (Aerial Digital sensor 80). In Figure 14, high association between the deformation patterns and the slipped areas can be observed, which confirm the potential Landslide do suffer deformation during the INSAR acquisition period (2014-2016). These displacement map can show a high consistency with the slipped area detected by aerial photo interpretation. This is recommended since the aerial image used for the slipped areas recognition is ADS80 images with $10 \mathrm{~cm}$ GSD, flight height $1000 \mathrm{~m}$. Therefore, the delineation of the slipped areas are based on rock avalanche, a groove and a crack.

It is good to emphasizethatInSARcanonlymeasure the deformation in the line-of-sight direction. If the true deformation of one Landslide is projected in the LOS direction, and the summation total of velocity (mm/year) data is close to zero, it is complicated to identified a potential landslide. Besides, it's recommended to involve ascending and descending track to have better ground deformation results, so in this study it's been used descending and ascending track (S1A/S1B IW SLC). Additionally, the deformation was analyze by multi-image framework recovered from October 2014 to December 2016 by using the InSAR technique, 26 Sentinel SAR images in Descending track and 17 Sentinel SAR images in Ascending track gathered over the Chosica city (witha relative temporal baseline of 3 years, and maximum relative normal baseline of less than 120m) werecoregister in a unique master (Sentinel descending orbit taken on November 05th, 2015). DEM was estimated with SRTM 30m. After DEM compensation, 25 differentialinterferogramsweregenerate. Then it's execute a join estimation of DEM errors, LOS velocities, and linear APS contributions. A joint estimation of both DEM errors and target velocity was then carried out. PSs are characterized by low phase residues. After DEM correction, PS target location is known to a fraction of meter depending on the local Signal to noise-ratio (SNR). The multi-interferogramfocusingstronglyreduces the impact of phase noise and residual atmospheric effects both on DEM errors and motion estimation.[6]. In geocoding step, it has chosen as a product coherence threshold: 0.80 , means that pixels with coherence values smaller than 0.80 couldn't be kept as persistent scaterrer. LOS displacement map's density points in descending orbit is about 133868 target points inside $170 \mathrm{~km} 2$ of study area. As already mentioned, the slipped areas is subject to very slow terrain motion as shown in table 8 and table 10 .

In other hand, the photointerpretation of the slipped areas with long-term evolution have identified, obtained temporal changes using aerial photo after landslide that occurred on 23 March, 2015(slipped areas shown on red polygon) and other landslide on January 15th 2017(slipped areas shown on orange polygon) shown in figure 30. Notice, Displacement map has obtained to detectedpotentiallandslide, InSAR data acquiredbefore Landslide (2017/01/15).

For better analysis of the displacement results, it has proceeded to separate each slipped area according to Ravine affected sectors and be able to appreciate velocity (mm/year) value deformation of each one (shown in Figure 14). The maximum velocity (mm/year) value of the selected sector is estimated as the deformation rate of the slipped areas (shown in table 5). 
As shown in Table 2 after rainstorm and landslides occurred in the study area, was active ravines Huampaní, Chacrasana, Santa Maria, San Antonio Pedregal, Quirio, Mariscal Castilla, California, Carossio, La Cantuta, Corrales, Santo Domingo; after analysis of displacement map, was detected considerable deformation in this areas, shows in the figure 14.

As shown in the table 8, the research's result of the velocity ( $\mathrm{mm} /$ year) and analyze of the damage in victims and main built structures, this study has foundLurigancho-Chosicalandslide has very slow displacement.

As illustrate in figure 14, the PSI displacement before the occurrence of the Chosicalandslide (2017/01/15). The red and orange polygons represent the slipped area detected using aerial photos, took after landslide (2015/03/23), and (2017/01/15) respectively. The black dotted polygon represents the landslide damage area. Remark that the deformation is in the LOS direction, the negative values (red) involve movements away from the sensor, and the positive values (blue) imply movements toward to the sensor. Notice that this Displacement map result obtained using Data from October, 2014 to December 2016 is to detected potential landslide, refer 2017 Landslide.

The displacement is produced every year, little by little motions, but when hard rains start make the situation worse, the origin land of the landslide appears in the top of mountain, where shown in the positive values (blue).

As can show in the figure 14 in the upper part, in the center there are some empty data, no permanent scaterrer(radar targets) becauseInSAR has some limitations due to temporal and geometrical decorrelation and atmospheric in homogeneities. Temporal decorrelationmakesInSARmeasurementsunfeasible over vegetated areas and where the electromagnetic profiles and/or the positions of the scatterers change with time within the resolution cell. Geometrical decorrelation limits the number of image pairs suitable for interferometric applications and prevents one from fully exploiting the data set available.[6]

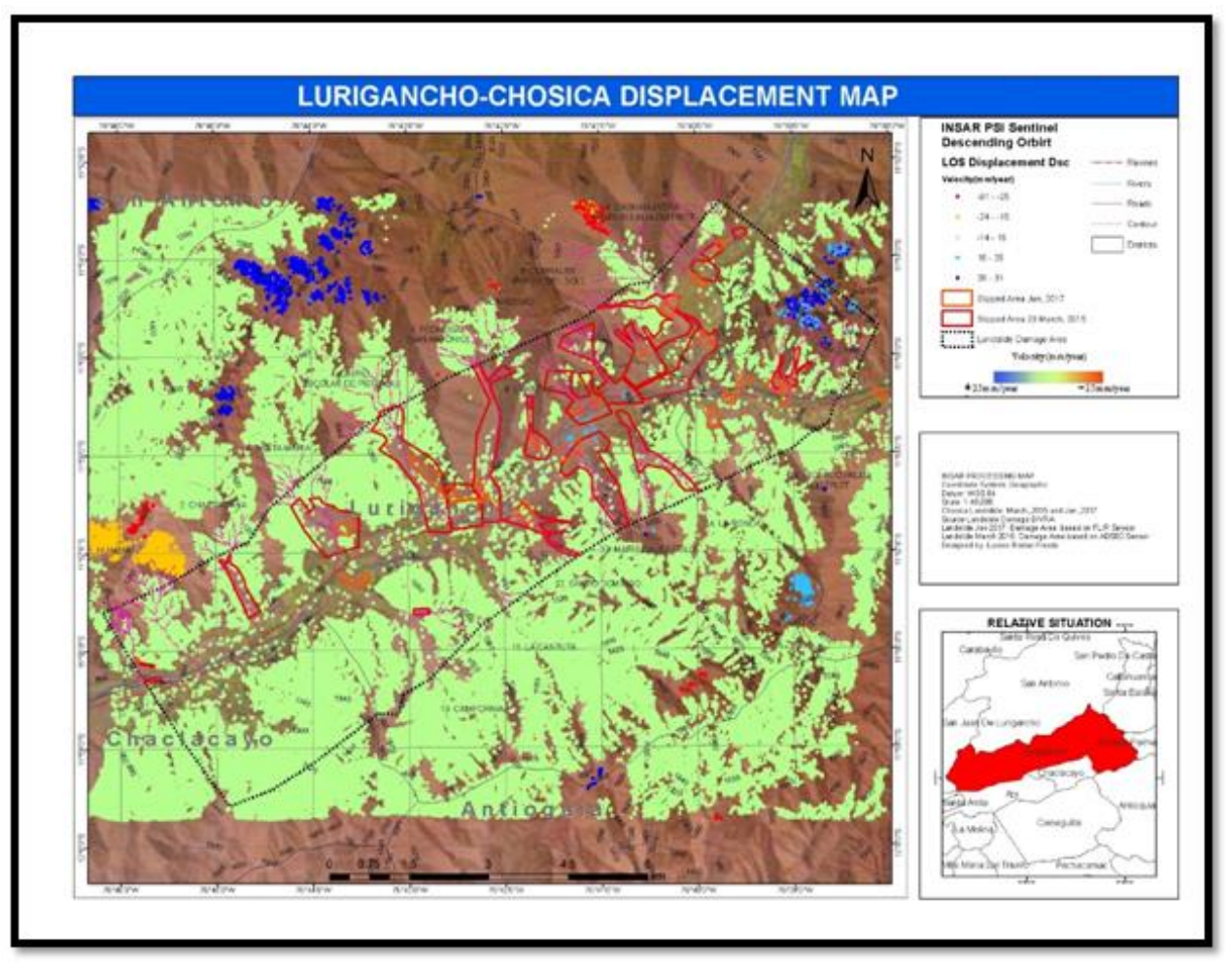

Figure 12: Displacement map-Descending Orbit 


\section{PSInSAR Method withascending orbit Sentinel 1A/B}

This research will process 17 scenes Sentinel images in ascending orbit, in order to compare PSInSAR descending results; and in other hand, it will process both orbit (Ascending and Descending) with the intention to find the behavior of the displacement. Table 10 shows the displacemtne values result of the study area.

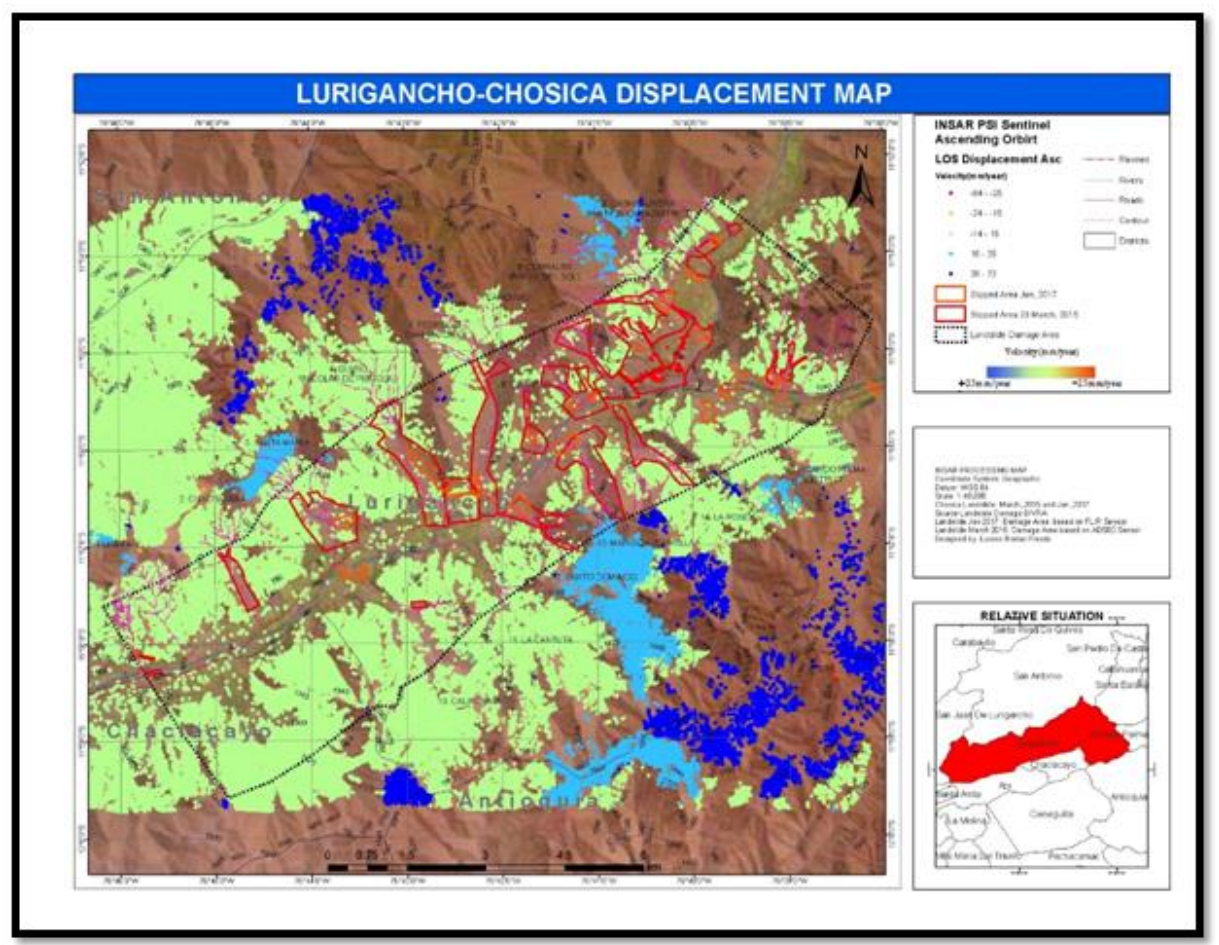

Figure 13 : 4Displacementmap-Ascending Orbit

East-West and Up-Down Displacement Velocity PSI (Ascending and descending orbit)

All the InSARtecniques can only measure the movements in Satellite Line of Sight (LOS) direction, this can be represented as vector formed from the ground target point to the satellite, the positive value in this direction means displacement toward the satellite, negative value means displacement away from satellite. Displacements Velocity in EastWest direction and Up-Down (vertical) direction can be obtained solving the equation 8, with ascending and descending orbit results the same area as input.

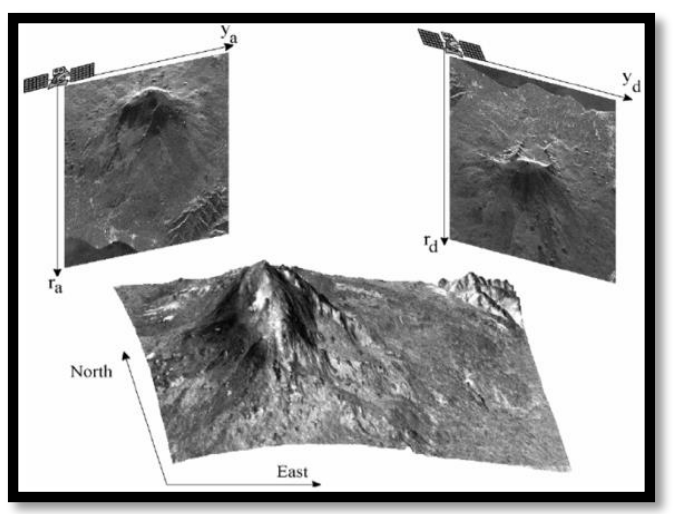

Figure 14: East-West and Up-Down Displacement Velocity 
After solve mathematically the equation 9, it has been obtained the following equations for displacement eastwest direction and displacement up-down direction. This equations have been calculated using tools of ArcGis-Python, through the following model builder. This equation was solved using 6 principal attributes (Vel_mm_y_, LOS_Az, LOS_In, Vel_mm_y1, LOS_Az_1, LOS_In_1).

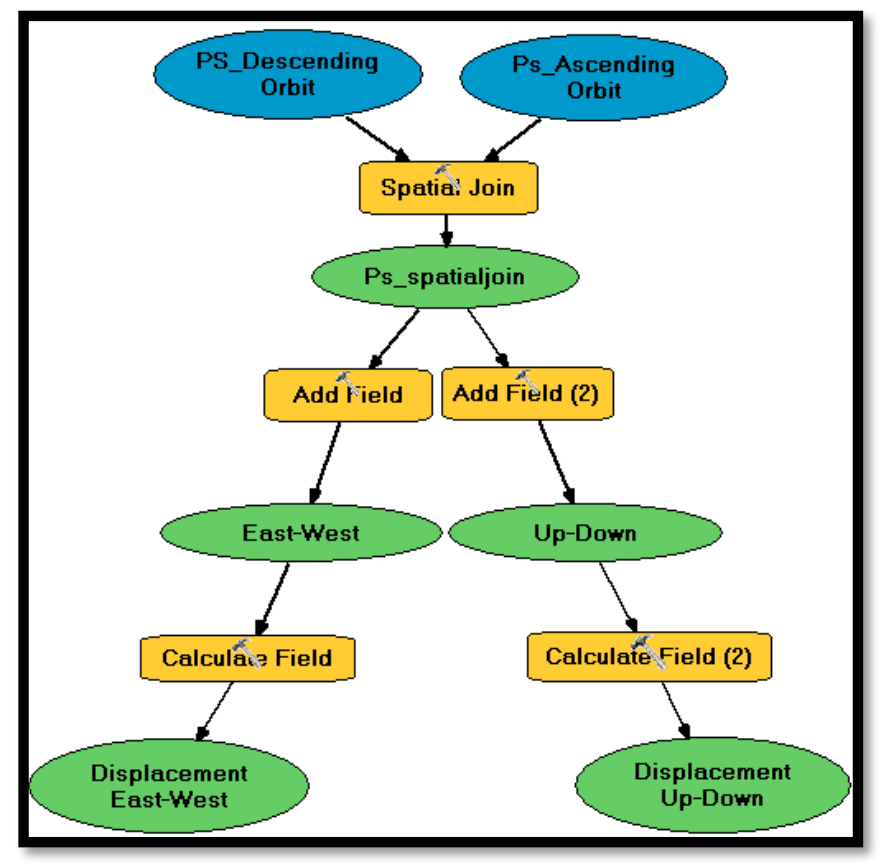

Figure 55 : Detection of East-West and Up-Down displacement, using Model builder-ArcGIS and equation (7, 8)

Afterapplying the model builder with Ascending and descending orbit described in the figure 17, East-West and Up Down displacement throw the followingresult: Lurigancho-Chosicalandslidewasaffected with $92.07 \mathrm{~mm} / \mathrm{year}$ westward, $41.13 \mathrm{~mm} /$ year eastward.

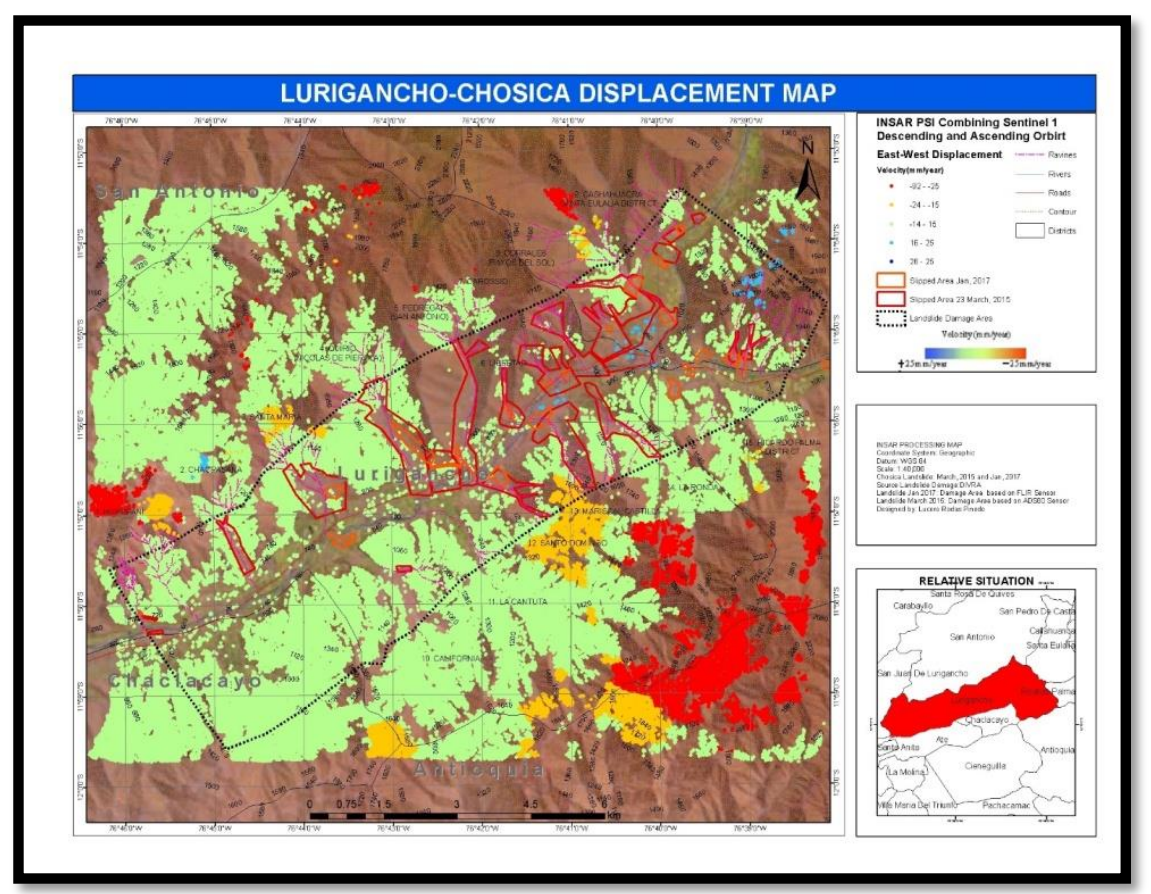

Figure 16 : East-West DisplacementmapjoinspatiallyAscending and DescendingOrbit 
As shown in the figure $(18,19)$; Chosica landslide was affected with $51.60 \mathrm{~mm} /$ year downward and $62.25 \mathrm{~mm} / \mathrm{year}$ upward. These outcomes found that the most affected areas of this landslide were in westward direction.

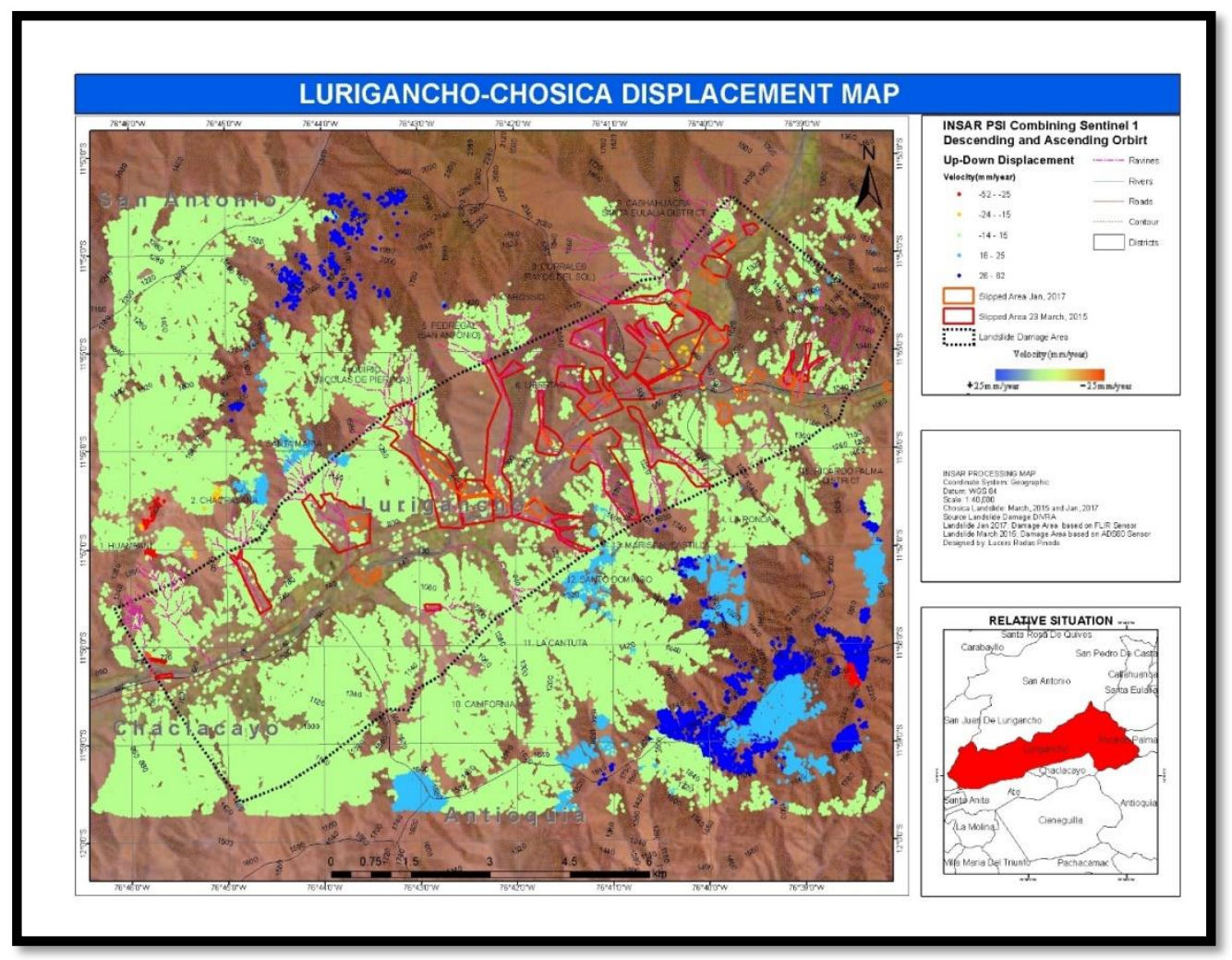

Figure 6 : Up-Down Displacementmapjoinspatially Ascending and Descending Orbit

\section{CONCLUSIONS}

- $\quad$ PS-InSARmethodisperfect to exploit a series of N SAR images (Interferometric stacking) in order to identify areas (pixels) that show coherent and consistent signal (displacement) over the time. PS have properties and characteristics that make them more or less suitable according to the typology of area of interest taken into account and the expected results.

- $\quad$ PS technique have been appliedeffectively in Lurigancho-Chosicalandslidewithcredible result taking account the affected area after landslide occurred, displacements detected expressed in velocity up to $9 \mathrm{~cm} / \mathrm{year}$ in east/west and $6 \mathrm{~cm}$ in up/down LOS direction have been perceived.

- Lurigancho-Chosicaisunstable and vulnerable area to landslide every year, so monitoring these hazard areas is national responsibility.

- The mostinestable areas in Lurigancho-Chosicawasfounded in Huanpani, Chacrasana, Santa Maria, Pedregal, Quirio and Santa Eulalia ravine, where the deformation rate in the source area(top of the mountain) of slipped area occurred in Huanpani, Chacrasana ravines was detected, the highest velocity reached $80.90 \mathrm{~mm} / \mathrm{year}$; and 30.71 in 
Quirio, Pedregal area.

- The deformation rate in slipped area occurred in Santa Eulalia, especially in Cashahuacra ravine wasdetected. The highest velocity reached $33.78 \mathrm{~mm} /$ year. Landslide 2017, affected district Santa Eulalia where 577 people was affected, 104 housing was affected, collapse and uninhabitable; $6.9 \mathrm{~km}$ of roads was affected and destroyed. The deformation rate in slipped area occurred in Cashahuacra ravine and surrounding of Santa Eulalia district have been detected as instable area, this area has been consider cause has some influence in Chosicadisaster and it's inside to the study area, next to Chosica district.

- Finally, It have been founded as principal movement in east-west direction with results $92.07 \mathrm{~mm} / \mathrm{year}$ westward, $41.13 \mathrm{~mm} /$ year eastward, $51.60 \mathrm{~mm} /$ year downward, and $62.25 \mathrm{~mm} /$ yearupward.

- This study can concludethat the InSARtechnologyisrecommended for long areas such as the Chosica's city, important due to its precision in millimeters, and low cost; at the same time, the free availability of sentinel-1 images are beneficial for anyresearcher.

- It may be more useful to compare the result of the displacement using the PSI InSAR technique with the GPS technique, which would provide and test the accuracy of the resultsobtained by PSI.

- Could be ideal to use a DEM whose spatial resolution in meters can reached less than 30m, nevertheless SRTM 30m canbeproductivelyexploited.

- It is advisable to plant more treesaround the streams of Chosica, whichwillminimize the damage and protect the population and, since there is not much vegetation, when the rains appear and find the land devoid of it, the terrain loses stability and it produces landslide

\section{REFERENCES}

1. Gutierrez, S. K., Morales, R. O., Díaz, O. D. L. C. A., \& Ruiz, E. P. (2018). Multispectral aerial image processing system for precision agriculture. Sistemas\&Telemática, 16(47), 45-58.

2. Villacorta Sandra; Nuñez Segundo; Huarez Cristian, F.L., Evaluacion geologica y consecuencias de los huaycos de Chosica del 23-03-15: Cronica de un desastre anunciado. [R] 2015: Lima. p. 68.

3. Activación de quebradas en el distrito de Lurigancho Chosica-Lima. [R] 2015, COEN, INDECI: Lima. p. 20.

4. Estrada.A, T.R., Precipitaciones pluviales en distritos de la provincia de Lima 2017 [R], COEN, INDECI: Lima. p. 29.

5. Ministry of Health, P., Sala Situacional de Emergencias por desastres en el Peru, enero 2017. [R] 2017. p. 39.

6. SARMAP, PS Tutorial. [G] p. 39. 
7. Ferretti, A., C. Prati, and F. Rocca, Permanent scatterers in SAR interferometry. [A] IEEE Transactions on Geoscience and Remote Sensing, 2001. 39(1): p. 8-20.

8. Fletcher, K., et al., InSAR principles : guidelines for SAR interferometry processing and interpretation.[S] 2007, Noordwijk, the Netherlands: ESA Publications Division, ESTEC.

9. Ferretti, A., C. Prati, and F. Rocca, Multibaseline InSAR DEM reconstruction: the wavelet approach. [J] IEEE Transactions on Geoscience and Remote Sensing, 1999. 37(2): p. 705-715. 